\title{
Prediction of three lipid derivatives for postoperative gastric cancer mortality: the Fujian prospective investigation of cancer (FIESTA) study
}

Dan $\mathrm{Hu}^{1+}$, Feng Peng ${ }^{2 \dagger}$, Xiandong Lin ${ }^{1}$, Gang Chen ${ }^{1}$, Binying Liang ${ }^{3}$, Ying Chen ${ }^{4}$, Chao Li ${ }^{1}$, Hejun Zhang ${ }^{1}$, Guohui Fan ${ }^{5}$, Guodong Xu ${ }^{5}$, Yan Xia ${ }^{1}$, Jinxiu Lin ${ }^{2}$, Xiongwei Zheng ${ }^{1 *}$ and Wenquan Niu ${ }^{*^{*}}$ (i)

\begin{abstract}
Background: As we previously reported, the presence of preoperative metabolic syndrome can predict the significant risk of gastric cancer mortality. As a further extension, we evaluated the prediction of three lipid derivatives generated from triglycerides (TG), total cholesterol (TC), high- and low-density lipoprotein cholesterol (HDLC and LDLC) at baseline for postoperative gastric cancer mortality by prospectively analysing 3012 patients. The three lipid derivatives included the ratio of TC minus HDLC to HDLC known as atherogenic index (Al), the ratio of TG to HDLC abbreviated as THR and the ratio of LDLC to HDLC abbreviated as LHR.
\end{abstract}

Methods: Gastric cancer patients who received gastrectomy between January 2000 and December 2010 were consecutively recruited from Fujian Cancer Hospital. Follow-up assessment was implemented annually before December 2015.

Results: Finally, there were 1331 deaths from gastric cancer and 1681 survivors, with a median follow-up time of 44.05 months. 3012 patients were evenly randomized into the derivation group and the validation group, and both groups were well balanced at baseline. Overall adjusted estimates in the derivation group were statistically significant for three lipid derivatives (hazard ratio [HR]: 1.20, 1.17 and 1.19 for Al, THR and LHR, respectively, all $P<0.001$ ), and were reproducible in the validation group. The risk prediction of three lipid derivatives was more obvious in males than females, in patients with tumor-node-metastasis stage I-II than stage III-IV, in patients with intestinal-type than diffusetype gastric cancer, in patients with normal weight than obesity, and in patients without hypertension than with hypertension, especially for AI and LHR, and all results were reproducible. Calibration and discrimination statistics showed good reclassification performance and predictive accuracy when separately adding three lipid derivatives to baseline risk model. A prognostic nomogram was accordingly built based on significant attributes to facilitate risk assessment, with a good prediction capability.

Conclusions: Our results indicate that preoperative lipid derivatives, especially Al and LHR, are powerful predictors of postoperative gastric cancer mortality, with more obvious prediction in patients of male gender or with tumor-nodemetastasis stage I-II or intestinal-type gastric cancer, and in the absence of obesity or hypertension before gastrectomy.

Keywords: The FIESTA study, Gastric cancer, Lipid derivative, Metabolic syndrome, Mortality, Prognosis

\footnotetext{
*Correspondence: agu1960@126.com; niuwenquan_shcn@163.com;

niuwenquan@zryhyy.com.cn

${ }^{\dagger}$ Dan Hu and Feng Peng contributed equally to this work.

'Department of Pathology, Fujian Cancer Hospital \& Fujian Medical University

Cancer Hospital, No.420 Fu Ma Road, Jin An District, Fuzhou 350014, Fujian,

China

${ }^{5}$ Institute of Clinical Medical Sciences, China-Japan Friendship Hospital, No.2

Yinghua East Street, Chao Yang District, Beijing 100029, China
}

(c) The Author(s). 2018 Open Access This article is distributed under the terms of the Creative Commons Attribution 4.0 International License (http://creativecommons.org/licenses/by/4.0/), which permits unrestricted use, distribution, and reproduction in any medium, provided you give appropriate credit to the original author(s) and the source, provide a link to the Creative Commons license, and indicate if changes were made. The Creative Commons Public Domain Dedication waiver (http://creativecommons.org/publicdomain/zero/1.0/) applies to the data made available in this article, unless otherwise stated. 


\section{Background}

Gastric cancer is one of the most common malignancies worldwide [1]. In China, national statistics show that gastric cancer is the second-leading cancer killer, and it took the lives of around 498,000 people in 2015 [2]. At present, early detection of gastric cancer currently poses a challenge, because most patients are asymptomatic at early stages, but progress to advanced stages once diagnosed [3, 4]. Thus far, surgery is a preferred choice to treat patients with resectable gastric cancer [5]. However, for some patients, the prognosis after gastrectomy or adjuvant treatment is unsatisfactory, with an overall 5 -year survival rate of less than $25 \%$ [6]. So, how to better predict the prognosis of postoperative gastric cancer patients thus far becomes a problem demanding prompt solutions [7, 8]. More recently, we, in an ongoing Fujian prospective investigation of cancer (FIESTA) study, analysed 3012 gastric cancer patients who were postoperatively monitored from 1.1 months to 183.3 months, and found that preoperative metabolic syndrome can significantly predict gastric cancer mortality after gastrectomy, to which systolic blood pressure, fasting blood glucose, triglyceride (TG) and high-density lipoprotein cholesterol (HDLC) contributed remarkably [9]. In this present study, to provide an in-depth evaluation of dyslipidaemia - an integral element of the metabolic syndrome, we generated three lipid derivatives from TG, total cholesterol (TC), HDLC and low-density lipoprotein cholesterol (LDLC) as prognostic predictors for gastric cancer mortality among 3012 patients after gastrectomy in the FIESTA database. The three lipid derivatives included the ratio of TC minus HDLC to HDLC known as atherogenic index (AI), the ratio of TG to HDLC abbreviated as THR and the ratio of LDLC to HDLC abbreviated as LHR, and they have been widely evaluated in association with cardiovascular diseases and the prediction capability is superior to individual lipids [10,11]. It remains uncertain, however, whether these lipid derivatives at baseline can effectively predict the prognosis of postoperative gastric cancer.

\section{Methods}

\section{The FIESTA study}

The FIESTA study is an ongoing exploration of preoperative factors for predicting disease-specific mortality of common digestive tract cancer, including sites at esophagus [12-15], stomach [9, 16] and colon and rectum [17-20]. The study proposal was approved by the Ethics Committee of Fujian Cancer Hospital. All patients gave written informed consent.

\section{Study patients}

Patients with gastric cancer were consecutively recruited from the Department of Thoracic Surgery, Fujian Cancer
Hospital between January 2000 and December 2010. Only patients who received total gastrectomy, distal partial gastrectomy or proximal partial gastrectomy based on the position and size of tumors were includable. Moreover, they reported no consanguinity and were of Han Chinese descent. Furthermore, they received gastrectomy for the first time, and had not received preoperative and postoperative chemotherapy or radiotherapy. Initially, a total of 3413 qualified patients were followed up.

\section{Follow-up assessment}

Postoperative patients who were discharged from Fujian Cancer Hospital were followed up annually prior to December 2015, unless lost to follow-up, dropped out of study or dead before that time. Follow-up assessment was conducted at the Out-Patient Department by contacting patients through phone calls or postal letters if they had missed appointments. The minimal follow-up was 1 month to avoid deaths from unclear surgical complications. Case-specific mortality from gastric cancer was the primary outcome. Survival time in months was calculated from the date of initial admission to the date of death or last follow-up visit, whichever occurred fist. Finally, 118 patients were lost to follow-up, 48 patients were monitored less than 1 month and 235 patients died of causes from non-gastric cancer, leaving 3012 patients in the present analysis. As of December 31, 2015, overall median follow-up time was 44.05 months (range: 1.1 months to 183.3 months), and 1331 deaths from gastric cancer occurred, leaving 1681 survivors. The minimal follow-up time of all patients was 5 years, which ensured sufficient power to predict postoperative survival at 5-year time point.

\section{Specimens}

Fasting venous blood specimens were drawn the day of receiving gastrectomy. Plasma TG, TC, and HDLC and LDLC were measured per standard procedures at the Clinical Laboratory of Fujian Cancer Hospital. Fasting blood glucose (FBG) was determined by an automated glucose-oxidase method.

Primary gastric cancer tissue and adjacent normal tissue specimens were surgically resected from all patients during the period of gastrectomy. All tissue samples were formalin-fixed, paraffin-embedded and frozen within $1 \mathrm{~h}$ after tumor removal.

\section{Baseline characteristics}

After signing informed consent, each patient was invited to complete a structured questionnaire covering information on date of birth, onset age of gastric cancer, gender, smoking status, drinking status and family history of cancer. Weight and height were measured at the time of admission when patients 
wore light clothing and no shoes. Body mass index (BMI) was calculated as the weight $(\mathrm{kg}) /$ height $(\mathrm{m})^{2}$. Blood pressure (BP) was measured on three occasions at roughly 5-min intervals using a mercury sphygmomanometer by certified nurses, while patients were in seated position per the standard protocol recommended by the American Heart Association [21]. Age of patients at the time of receiving gastrectomy was recorded. Smoking status was categorized into never smoking and ever (former/current) smoking. Drinking status was categorized into never drinking and ever (former/ current) drinking. Family cancer history was reported if one or more of affected relatives within three generations who suffered from malignancies except non-melanoma skin cancer.

Obesity was defined as BMI $\geq 25 \mathrm{~kg} / \mathrm{m}^{2}$. Hypertension was defined as systolic BP $\geq 140 \mathrm{mmHg}$ or diastolic BP $\geq 90 \mathrm{mmHg}$ or use of antihypertensive medications. Diabetes was defined as $\mathrm{FBG} \geq 7.0 \mathrm{mmol} / \mathrm{L}$.

\section{Clinicopathologic characteristics}

Clinicopathologic characteristics were elicited from medical charts and pathological reports of patients, including tumor-node-metastasis (TNM) stage (I-IV) [22], tumor size (in centimeters), depth of invasion (T1-T4), regional lymph node metastasis (N0-N3), distant metastasis (M0 and M1), Lauren's classification (intestinal type and diffuse type), number of lymph node metastasis (LNM) and tumor embolus.

\section{Statistics}

To derive a reproducible estimate, all 3012 patients were evenly randomized into the derivation group and the validation group. Gastric cancer mortality was calculated in both groups. Continuous variables were presented as mean (standard deviation or SD) or median (interquartile range), and their comparisons between the derivation and validation groups were implemented with the t-test or the Mann-Whitney test if appropriate. Categorical variables were presented as percentage (count) and compared with the $[\mathrm{Chi}]^{2}$ test. Weibull proportional hazards regression analysis was performed to estimate hazard ratio with its $95 \%$ confidence interval ( $95 \% \mathrm{CI}$ ) of each lipid derivative for gastric cancer mortality with and without considering confounders. For binary lipid derivatives, Kaplan-Meier curves were used to display changes of cumulative survival rates against follow-up time, and Log-rank tests were used to compare differences in median survival time (MST).

Predictive accuracy was evaluated with discrimination and calibration statistics. Discrimination statistics included Harrell C-statistic to see whether the addition of individual lipid derivatives can differentiate among patients who died from gastric cancer or survived. Calibration statistics included Akaike information criterion (AIC) and Bayesian information criterion (BIC), and the $-2 \log$ likelihood ratio tests were used to assess how closely prediction probability by adding lipid derivatives reflected the actual observed risk and global fit of modified risk model.

In addition, net benefit gained by adding individual lipid derivatives to traditional risk model was displayed as a curve in decision curve analysis [23]. The $\mathrm{X}$-axis of this curve represents thresholds for gastric cancer mortality, and the Y-axis represents net benefits hinged on different thresholds. A higher net benefit is shown if the "model" curve is farther away from solid curve line when assuming all gastric cancer mortality and dotted horizontal line when assuming none gastric cancer mortality. The farthest the curve is, the highest the net benefit is.

Finally, a prognostic nomogram displaying 3-year, 5 -year and 10-year gastric cancer mortality was constructed for clinical application among all study patients. The attributes in nomogram were selected from baseline demographic, clinical and clinicopathologic variables that were significantly associated with gastric cancer mortality besides three lipid derivatives. The predictive accuracy and discriminative capability of this nomogram were assessed by the concordance index (C-index) and calibration curves. The $\mathrm{C}$-index ranges from 0.0 to 1.0 , with the higher value indicating a higher accuracy. It is generally accepted that the C-index of less than 0.7 suggests no improvement in model performance [24]. In calibration curve, the 45-degree line represents optimal predictions, and it illustrates how far the predicted probabilities of this nomogram are from actual observations. The prognostic nomogram was generated by the regression modeling strategies (RMS) package (https://cran.r-project.org/web/ packages/rms/index.html) in the R-language (version 3.3.3).

Unless otherwise stated, the STATA/SE software (version 14.0, StataCorp, TX, USA) was employed for data management and statistical analyses. $P$ value less than 0.05 was considered statistically significant.

\section{Results \\ Baseline characteristics}

Baseline demographic, clinical and clinicopathologic characteristics were compared between the derivation and validation groups, as shown in Table 1. Both groups had an equal number of study patients. All characteristics were comparable between the two groups, except gender composition $(P=0.051)$.

\section{Risk estimates}

The risk estimates of three lipid derivatives were calculated based on per SD increment of AI (SD: 2.12), THR 
Table 1 Comparisons of baseline demographic, clinical and clinicopathologic characteristics between the derivation and validation groups

\begin{tabular}{|c|c|c|c|}
\hline Characteristics & Derivation group & Validation group & $P$ \\
\hline Number & 1506 & 1506 & \\
\hline Age (years) & $58.47(11.09)$ & $58.76(11.30)$ & 0.503 \\
\hline Males & $72.78 \%(1096)$ & $75.9 \%(1143)$ & 0.051 \\
\hline Ever smokers & $18.39 \%(277)$ & $18.53 \%(279)$ & 0.925 \\
\hline Ever drinkers & $5.84 \%(88)$ & $5.31 \%(80)$ & 0.525 \\
\hline Family cancer history (+) & $8.43 \%(127)$ & $8.7 \%(131)$ & 0.795 \\
\hline Body mass index $\left(\mathrm{kg} / \mathrm{m}^{2}\right)$ & $22.72(3.14)$ & $22.91(3.01)$ & 0.104 \\
\hline Systolic blood pressure (mmHg) & $124.16(19.03)$ & $124.84(19.92)$ & 0.353 \\
\hline Diastolic blood pressure (mmHg) & $76.85(11.37)$ & $77.43(11.51)$ & 0.179 \\
\hline Fasting blood glucose (mmol/L) & $6.21(2.48)$ & $6.19(2.43)$ & 0.853 \\
\hline $\mathrm{Al}$ & $3.95(2.28)$ & $3.88(1.93)$ & 0.209 \\
\hline THR & $1.41(1.47)$ & $1.36(1.27)$ & 0.339 \\
\hline LHR & $3.30(1.79)$ & $3.28(1.56)$ & 0.505 \\
\hline TNM stage & & & 0.122 \\
\hline I & $12.44 \%(177)$ & $11.76 \%(167)$ & \\
\hline$\|$ & $15.95 \%(227)$ & $13.87 \%(197)$ & \\
\hline III & $54.67 \%(778)$ & $59.01 \%(838)$ & \\
\hline IV & $16.94 \%(241)$ & $15.35 \%(218)$ & \\
\hline Lauren's classification & & & 0.551 \\
\hline Intestinal type & $39.77 \%(552)$ & $38.04 \%(528)$ & \\
\hline Diffuse type & $60.23 \%(836)$ & $61.96 \%(860)$ & \\
\hline Tumor embolus & & & 0.976 \\
\hline Positive & $39.01 \%(541)$ & $38.95 \%(541)$ & \\
\hline Negative & $60.99 \%(846)$ & $61.05 \%(848)$ & \\
\hline Tumor size $(\mathrm{cm})$ & $5.55(2.85) ; 5(3.5,7)$ & $5.66(3.13) ; 5(3.5,7)$ & 0.337 \\
\hline Number of lymph node metastasis & $5.60(6.91) ; 3(0,8)$ & $5.70(7.10) ; 3(0,8)$ & 0.724 \\
\hline
\end{tabular}

Data are represented as mean (standard deviation) or median (interquartile range) or percentage (count)

Abbreviations: Al atherogenic index, THR the triglyceride to high-density lipoprotein cholesterol ratio, LHR the low-density lipoprotein cholesterol to high-density lipoprotein cholesterol ratio, TNM tumor-node-metastasis

(SD: 1.38) and LHR (SD: 1.68) among all study patients. Overall and stratified risk estimates of three lipid derivatives in both groups for gastric cancer mortality are summarized in Table 2. Relative to unadjusted overall estimates, consideration of possible confounding factors attenuated risk estimates for all three lipid derivatives in both groups, yet still being remarkably significant.

By gender, the risk prediction of three lipid derivatives was markedly corroborated in male patients compared with female patients in both derivation and validation groups, especially for AI (HR, 95\% CI, P: 1.27, 1.17-1.38, $<0.001$ in the derivation group and 1.30, 1.18-1.43, < 0.001 in the validation group) and $\operatorname{LHR}(1.25,1.15-1.36$, $<0.001$ in the derivation group and 1.26, 1.16-1.38, < 0.001 in the validation group). By smoking status, none or marginal significance was detected in ever smokers, while there was significant association between baseline three lipid derivatives and postoperative gastric cancer mortality, and risk estimates were comparable between both groups. By TNM stage, three lipid derivatives can better predict the significant risk of gastric cancer mortality in patients with stage I-II than those with stage III-IV, especially for AI (HR, 95\% CI, P: 1.59, 1.34-1.87, $<0.001$ versus $1.19,1.13-1.27,<0.001$ in the derivation group) and $\operatorname{LHR}(1.67,1.38-2.01,<0.001$ versus 1.17, $1.10-1.23,<0.001$ in the derivation group), and the prediction was reproducible in the validation group. By Lauren's classification, there was significant prediction for three lipid derivatives, especially for intestinal-type gastric cancer, and the results were consistently reproduced in 


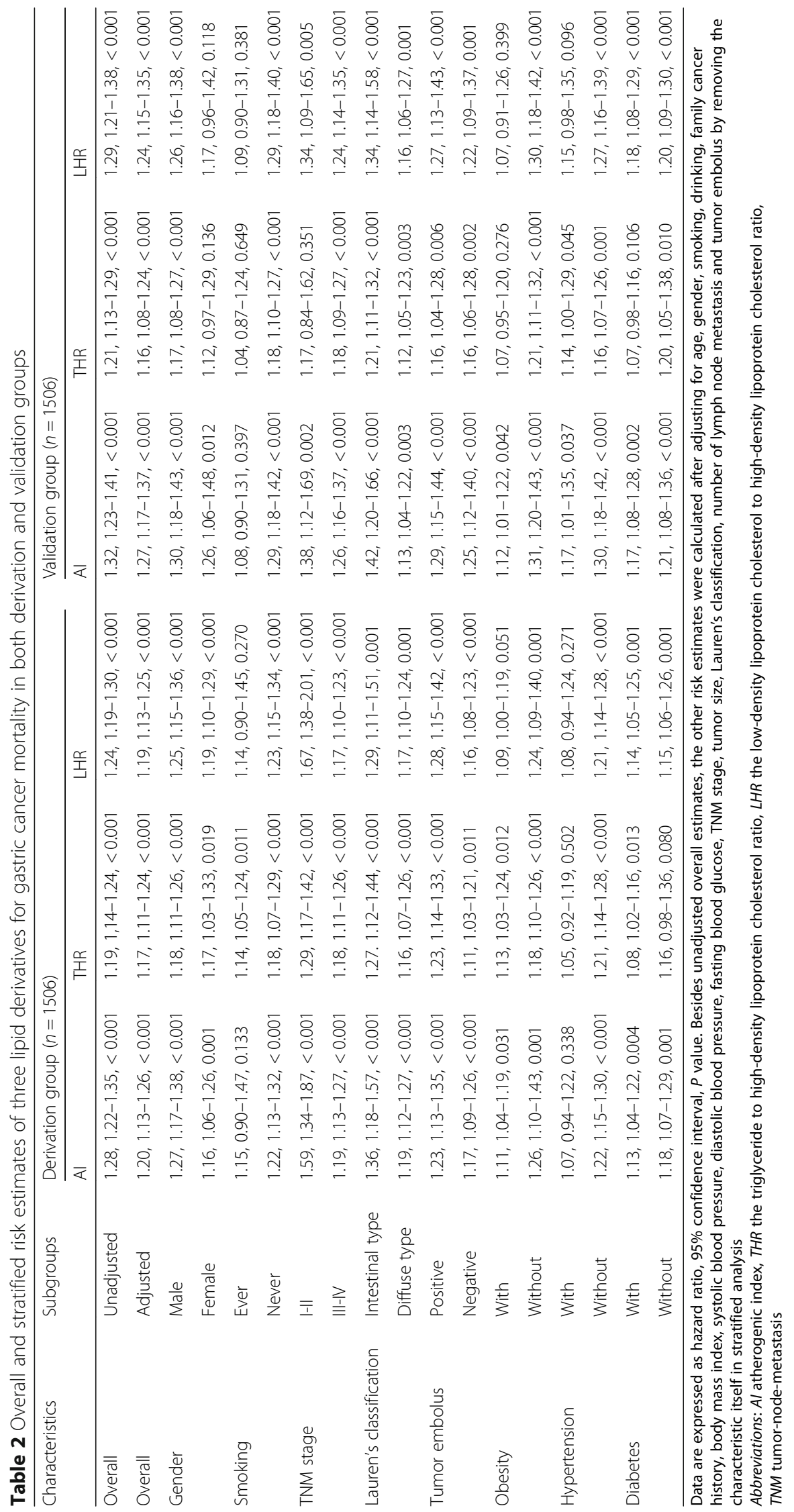


the validation group. By tumor embolus, the risk estimates were remarkably significant but slightly stronger in patients with positive embolus than in patients with negative embolus in both groups.

By obesity, the association of three baseline lipid derivatives, AI (HR, 95\% CI, P: 1.26, 1.10-1.43, 0.001 versus $1.11,1.04-1.19,0.031$ in the derivation group) and LHR $(1.24,1.09-1.40,0.001$ versus $1.09,1.00-1.19,0.051)$ with gastric cancer mortality was stronger in normal weight patients than in obese patients, and the same trend was observed in the validation group. By hypertension, risk was significant in patients without hypertension, and its magnitude was stronger than patients with hypertension in both groups. By diabetes, only $\mathrm{AI}$ and LHR exhibited a significant association with gastric cancer mortality, which was slightly reinforced in patients without diabetes, and the derived results were reproduced in the validation group (Table 2).

Although the majority of comparisons were reproducible between the derivation group and the validation group, there were some exceptions, for example, the risk prediction of THR and LHR for gastric cancer prognosis in female patients was significant in the derivation (HR, 95\% CI, P: 1.17, 1.03-1.33, 0.019 and $1.19,1.10-1.29,<0.001)$ but not in the validation group (1.12, $0.97-1.29,0.136$ and 1.17, 0.96-1.42, 0.118 ), possibly due to insufficient statistical power as there were respectively 410 (27.22\%) and 363 (24.1\%) female patients involved in the derivation and validation groups.

\section{Kaplan-Meier survival analysis}

The median values of AI, THR and LHR were 3.41, 0.97 and 2.89 among all study patients, respectively, and they were used as binary cut-off thresholds in Kaplan-Meier survival curves (Fig. 1). Patients with lower levels of AI (Fig. 1a and b), THR (Fig. 1c and d) and LHR (Fig. 1e and f) separately had better survival rates than those with the corresponding higher levels in both derivation and validation groups. In addition, MST was significantly longer in patients with lower levels of AI, THR and LHR than those with the corresponding higher levels (Log-rank test $P<0.0001$ for all comparisons) in both groups.

\section{Calibration and discrimination}

Calibration and discrimination abilities for the addition of individual lipid derivatives to the baseline risk model in predicting gastric cancer mortality are presented in Table 3. Both AIC and BIC statistics were reduced by more than 10 after the addition of AI and LHR to the baseline risk model in both derivation and validation groups. No material changes in both statistics were noted for THR. In addition, the $-2 \log$ likelihood ratio test was markedly significant for AI and LHR in both groups, indicating that $\mathrm{AI}$ and LHR were indeed a part of true model and carried a better fit, while there was only marginal significance for THR.

As indicted by the Harrell's C-statistic, baseline risk model and modified models by separately adding three individual lipid derivatives had better predictive accuracy in both derivation and validation groups.

\section{Decision curve analysis}

Based on decision curve analysis, gained usefulness of individual lipid derivatives over the baseline risk model is presented in Fig. 2. Gained net benefits by adding AI (Fig. 2a and b), THR (Fig. 2c and d) and LHR (Fig. 2e and $\mathrm{f}$ ) were higher than that of the baseline risk model in both derivation and validation groups.

\section{Prognostic nomogram}

A nomogram was depicted to predict 3-year, 5-year and 10-year individualized absolute risk for gastric cancer mortality based on significant attributes among all study patients (Fig. 3). Significant attributes were selected from baseline demographic, clinical and clinicopathologic characteristics by the Weibull proportional hazards model, including age, systolic BP, diastolic BP, FBG, TNM stage, Lauren's classification, tumor size, number of LNM, BMI, AI, THR and LHR (all $P<0.05$ ). Predictive accuracy of this nomogram was good, with the C-index of being 0.773. Calibration curves for 3-year, 5-year and 10-year survival prediction indicated good agreement between predicted probabilities by this nomogram and actual observations (Additional file 1: Figure S1).

\section{Discussion}

Extending our previous findings on metabolic syndrome and gastric cancer [9], we evaluated the prediction of three lipid derivatives for the prognosis of postoperative gastric cancer. Importantly, our findings indicate that lipid derivatives, especially AI and LHR, are powerful predictors of gastric cancer mortality, and the prediction is more obvious in patients of male gender or with TNM stage I-II or intestinal-type gastric cancer, and in the absence of obesity or hypertension before gastrectomy. A revisit to derived components of the metabolic syndrome would be an essential step toward better understanding of metabolomics in the pathophysiology of gastric cancer, and facilitate the development of new therapeutic targets.

Recently, Xiao and Zhou have written an excellent review about the metabolism and metabolomics of gastric cancer, and they underscored the close relevance between metabolic changes and gastric carcinogenesis, implicating the metabolism-based antitumor therapies in future clinical practice [25]. In this context, some studies 


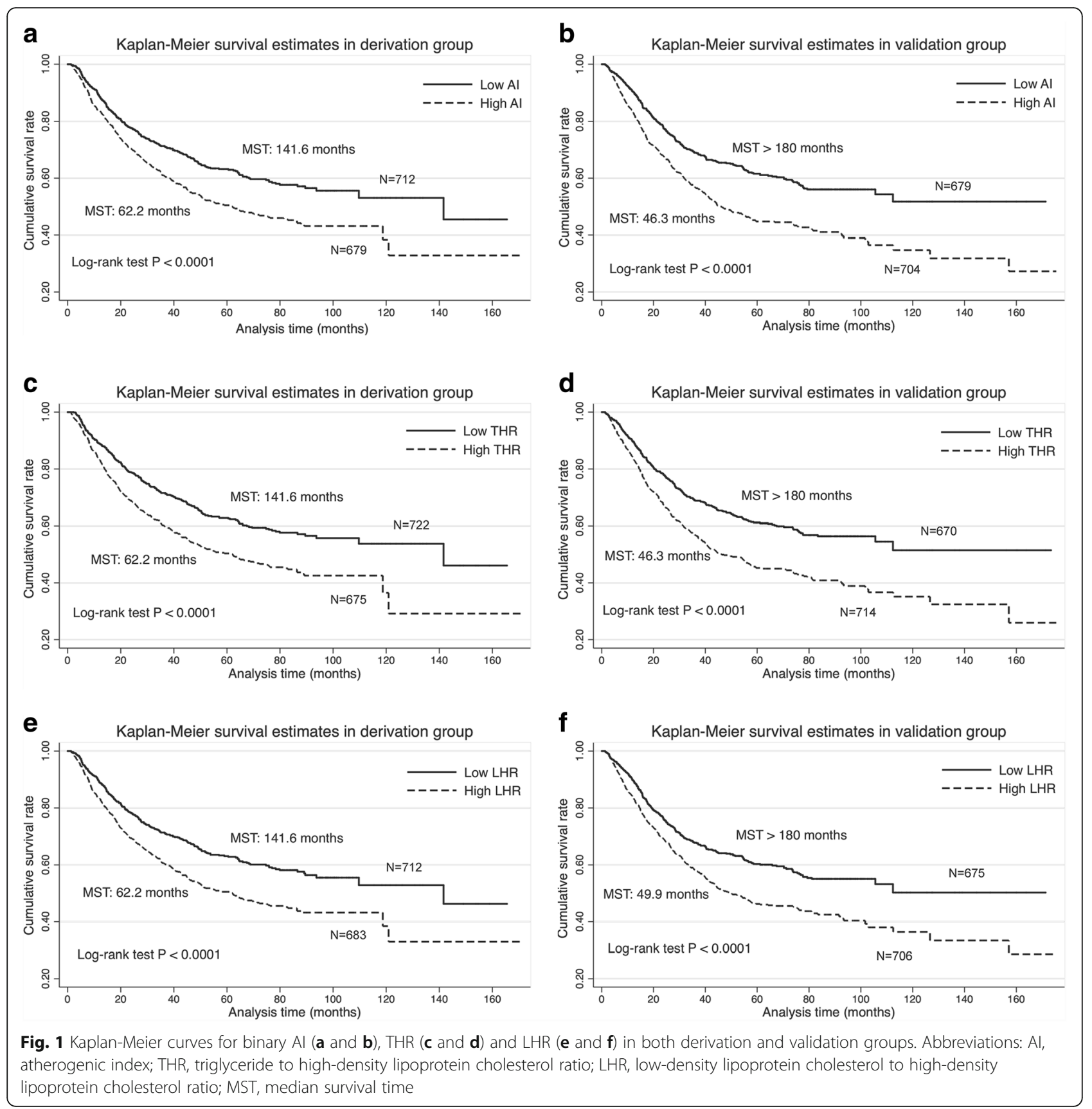

have investigated the influence of the metabolic syndrome and its components on the risk of developing gastric cancer [26-29]. However, less research on the prediction of coexisting metabolism syndrome for postoperative survival of gastric cancer has been done so far. For example, Wei and colleagues reported that gastric cancer patients complicated with the metabolism syndrome had improved tumor cell differentiation and had a better chance of postoperative survival [30]. Another study by Kim and colleagues revealed that coexistence of the metabolic syndrome can increase the risk of gastric cancer mortality after receiving gastrectomy [31], as consolidated by our recent FIESTA study in a prospective analysis of 3012 gastric cancer patients over a 15-year follow-up period [9]. Hence, exhaustive research is still needed to address these inconsistencies to enable precise clinical translation.

It is well known that the metabolic syndrome is the collective term given to a group of risk factors that evoke cardiovascular disease and diabetes, and abnormalities in 
Table 3 Predictive accuracy of baseline risk model and the addition of three individual lipid derivatives for gastric cancer mortality in both derivation and validation groups

\begin{tabular}{|c|c|c|c|c|c|c|c|c|}
\hline \multirow[t]{2}{*}{ Statistics } & \multicolumn{4}{|c|}{ Derivation group $(n=1506)$} & \multicolumn{4}{|c|}{ Validation group $(n=1506)$} \\
\hline & BR-Model (BRM) & BRM plus $\mathrm{Al}$ & BRM plus THR & BRM plus LHR & BR-Model (BM) & BRM plus $\mathrm{Al}$ & BRM plus THR & BRM plus LHR \\
\hline$\overline{\mathrm{AIC}}$ & 2476 & 2461 & 2473 & 2458 & 2604 & 2584 & 2599 & 2586 \\
\hline $\mathrm{BIC}$ & 2532 & 2523 & 2531 & 2519 & 2660 & 2645 & 2660 & 2647 \\
\hline LR test: $\left[\right.$ Chi] ${ }^{2}$ & Reference & 16.26 & 4.9 & 17.85 & Reference & 12.58 & 5.34 & 12.55 \\
\hline LR test: $P$ & Reference & 0.0001 & 0.0269 & $<0.0001$ & Reference & 0.0004 & 0.0208 & 0.0004 \\
\hline Harrell's C & 0.7662 & 0.7693 & 0.7679 & 0.7697 & 0.7541 & 0.7587 & 0.7555 & 0.7579 \\
\hline
\end{tabular}

BR-Model included age, gender, smoking, drinking, family cancer history, body mass index, systolic blood pressure, diastolic blood pressure, fasting blood glucose, tumor-node-metastasis stage, tumor size, Lauren's classification, number of lymph node metastasis and tumor embolus

Abbreviations: BR-Model (BRM) baseline risk model (BRM), Al atherogenic index, THR the triglyceride to high-density lipoprotein cholesterol ratio, $L H R$ the lowdensity lipoprotein cholesterol to high-density lipoprotein cholesterol ratio, AIC Akaike information criterion, BIC Bayesian information criteria, $L R$ test likelihood ratio test

these factors will aid clinical management of the metabolic syndrome as an efficient therapeutic strategy to improve survival outcomes of gastric cancer patients [32, 33]. In particular, dyslipidemia has been widely recognized as a diagnostic tool for the metabolic syndrome [34]. According to the criteria proposed by the Chinese Diabetes Society (2004) [35], dyslipidaemia is contingent on a simple assembly of TG and HDLC, and it disregards the other two blood lipids - TC and LDLC. To provide an in-depth evaluation of dyslipidemia, we developed three ratio derivatives based on the four blood lipids, and plenty of studies have pointed out these three lipid derivatives as more effective predictors of cardiovascular diseases than individual lipids $[10,11]$. However, it is rarely reported about the predictive role of these lipid derivatives in the development and progression of gastric cancer. The present study attempted to fill the gap on this topic by revisiting the FIESTA database [9, 16] to evaluate the long-term prediction of three lipid derivatives at baseline for the prognosis of 3012 gastric cancer patients after gastrectomy. We importantly found that three lipid derivatives, especially AI and LHR, were powerful predictors of gastric cancer mortality, and the predictive performance was augmented in male or early stage patients or with coexistent obesity or hypertension at baseline. From a clinical perspective, our findings were biologically meaningful. It has been proposed that lipid abnormalities, especially reduced HDLC, play an important role in carcinogenesis through antioxidant and anti-inflammatory properties [36, 37]. HDLC was reported to modulate angiogenesis, a critical biological process that is altered during carcinogenesis, in a multifunctional manner, depending on pathophysiological context [38]. In addition, other potential biologic mechanisms might involve the changes of endogenous hormones such as sex steroids associated with obesity and the contribution of obesity to gastric carcinogenesis [39, 40]. Exploring the underlying molecular mechanisms of lipid abnormalities in gastric carcinogenesis is beyond the scope of our present study but certainly requires further investigation. So, we propose that clinical management of lipid abnormalities, especially HDLC, can help prolong survival and improve quality of life for patients with resectable gastric cancer.

From a statistical perspective, our findings are reproducible (with few exceptions), reliable and applicable. In fact, we adopted a two-phase sampling design by evenly randomizing study patients into the derivation group and the validation group. Of note, all effect-size estimates generated in the derivation group were consistently reproduced in the validation group, underscoring the robustness of these lipid derivatives. In addition, our findings have been proven to bear high reliability, as multiple calibration and discrimination statistics consistently indicated that adding individual lipid derivatives to baseline risk model had better classification performance and predictive accuracy. Furthermore, in view of strong predictive power of three lipid derivatives, we established a prognostic nomogram as a calculator to predict 3-year, 5-year and 10-year individualized absolute risk, and this nomogram had good predictive accuracy, as reflected by both $\mathrm{C}$-index and calibration curves. Hence, our findings can be easily applied for risk assessment in routine clinical practice.

Finally, several limitations should be acknowledged in this study. First, this study was carried out in a mono-centre, and the findings could be generalizable pending consistently validated in other studies and cohorts. Second, the effects of lipid-lowering agents on the prognosis of gastric cancer patients remained unexplored, as such data were currently lacking, which might generate a systematic bias and unaccounted 

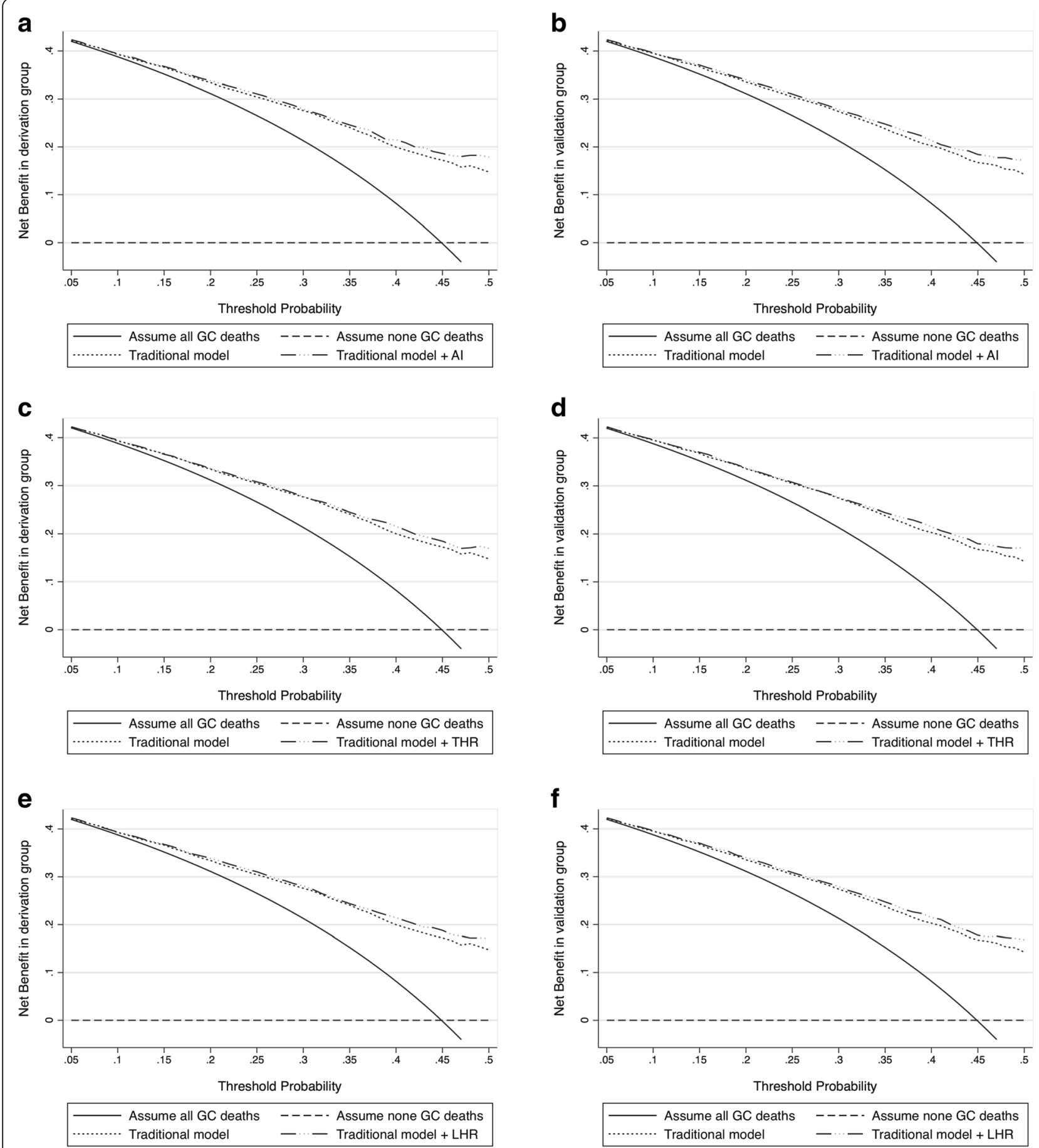

Fig. 2 Decision curves for baseline risk model (termed traditional model) and the addition of Al (a and $\mathbf{b}$ ), THR (c and $\mathbf{d}$ ) and LHR (e and $\mathbf{f}$ ) in both derivation and validation groups. Abbreviations: Al, atherogenic index; THR, triglyceride to high-density lipoprotein cholesterol ratio; LHR, low-density lipoprotein cholesterol to high-density lipoprotein cholesterol ratio; GC, gastric cancer. Baseline risk model included age, gender, smoking, drinking, family cancer history, body mass index, systolic blood pressure, diastolic blood pressure, fasting blood glucose, tumor-nodemetastasis stage, tumor size, Lauren's classification, number of lymph node metastasis and tumor embolus

residual confounding. Third, all study patients were recruited between January 2000 and December 2010, and during such a long period, remarkable advances in surgical techniques might introduce a possible bias.
Fourth, our findings were based on only gastric cancer patients who received gastrectomy, and thereby cannot be extrapolated to the general patient populations. Nonetheless, not only does lipid-lowering treatment 


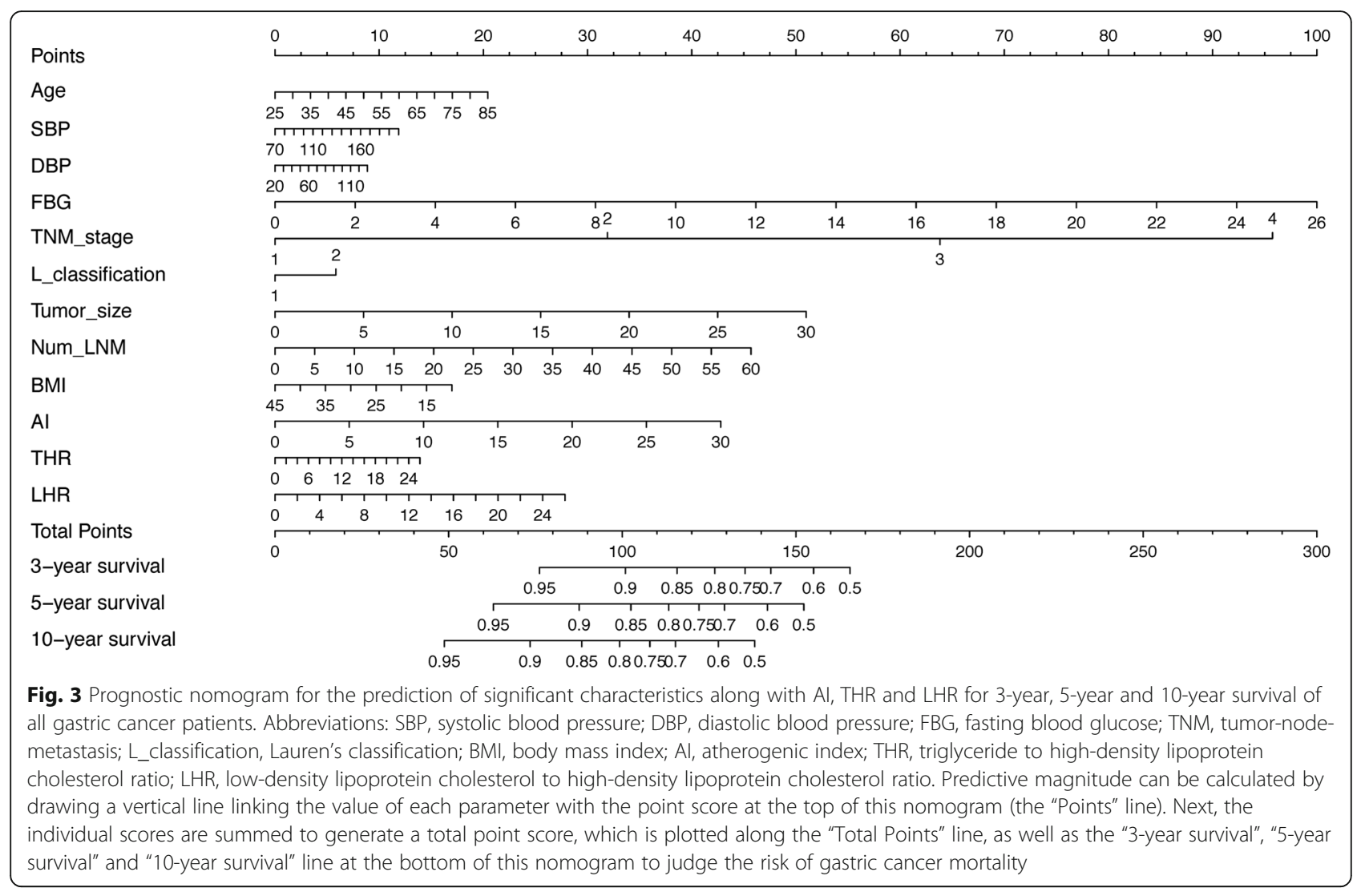

have significant clinical implications in gastric cancer patients, but it is necessary for future continued investigations to unravel the molecular mechanisms linking dyslipidaemia with poor prognosis for precise therapeutic intervention and finding additional drug targets, which will have considerable public health significance.

\section{Conclusions}

Based on an in-depth analysis, our findings indicate that preoperative lipid derivatives, especially AI and LHR, are powerful predictors of gastric cancer mortality, and the prediction is more obvious in patients of male gender or with TNM stage I-II or intestinal-type gastric cancer, and in the absence of obesity or hypertension before gastrectomy. For practical reasons, an increased understanding of lipid abnormalities in gastric carcinogenesis can provide the foundation to facilitate the development of therapeutic agents that may identify novel applications for future clinical testing in postoperative gastric cancer patients.

\section{Additional file}

Additional file 1: Figure S1. Calibration curves for predicting the risk of gastric cancer mortality at 3 years (A), 5 years (B) and 10 years (C) among all gastric cancer patients. Nomogram-predicted probability of overall survival is plotted on the $\mathrm{X}$-axis, and actual overall survival is plotted on the $\mathrm{Y}$-axis. (PDF $79 \mathrm{~kb}$ )

\section{Abbreviations}

95\% Cl: 95\% confidence interval; Al: Atherogenic index; AlC: Akaike information criterion; BIC: Bayesian information criterion; BMI: Body mass index; BP: Blood pressure; C-index: Concordance index; FBG: Fasting blood glucose; FIESTA: Fujian Prospective Investigation of Cancer; HDLC: Highdensity lipoprotein cholesterol; HR: Hazard ratio; LDLC: Low-density lipoprotein cholesterol; LHR: The ratio of LDLC to HDLC; LNM: Lymph node metastasis; MST: Median survival time; RMS: Regression modeling strategies; SD: Standard deviation; TC: Total cholesterol; TG: Triglycerides; THR: The ratio of TG to HDLC; TNM: Tumor-node-metastasis

\section{Acknowledgements}

We thank our colleagues over the years at Fujian Cancer Hospital - particularly Xiaohui Chen, Yuzhen Zheng, Qingfeng Zheng, Shuoyan Liu, Zhilian She, Kunshou Zhu, Weidong Zang, Weizhong Ruan, Weimin Fang, Lin Li, Mingqiang Chen, Derong Zhang, Shaofeng Lin, Shunjin Chen, Yigui Chen and Guohong Zhao for performing the surgery, Yanni Gao, Zhenzhou Xiao, Su Lin, Xuehong Liao, Wenhui Jiang, Jieqiong Lin, Xinjing Li, Yi Shi, Xiaojiang Wang, Shanfeng Jin, Hongfei Wang, Wucheng Shen, Weifeng Zhu, Xiaowen Cai, Baozhen Chen, Tongmei Chen, Xueyan Chen and Lifang Chen for collecting the blood/tissue samples and performing the follow-up investigations.

\section{Funding}

This study was financially supported by the Natural Science Foundation of Fujian Province (grants no. 2016 J01503, 2016 J01508), the Training Project for Young and Middle-Aged Core Talents of Health System of Fujian Province (grants no. 2015-ZQN-JC-7) and the Ministry of Health P.R. China (WKJ20162-05). The funders of this study had no role in the design of the study and collection, analysis, and interpretation of data and in writing the manuscript. 


\section{Availability of data and materials}

The datasets used and/or analyzed during the current study are available from the corresponding author on reasonable request.

\section{Authors' contributions}

$\mathrm{DH}, \mathrm{XZ}, \mathrm{WN}$ planned and designed the study, and directed its implementation; $D H, F P, J L, X Z$ drafted the protocol; $G C, Y C, X L, B L, C L, H Z$, YX obtained statutory and ethics approvals; $D H, X L, G C, B L, Y C, C L, H Z, Y X$ contributed to data acquisition; WN, FP, GF, GX conducted statistical analyses; WN, DH, FP, XZ had access to all raw data; DH, FP, WN did the data preparation and quality control; DH, FP, JL, WN wrote and revised the manuscript. All authors read and approved the final manuscript prior to submission.

\section{Ethics approval and consent to participate}

Ethical approval was obtained from the ethics committee of Fujian Cancer Hospital. All patients gave written informed consent for the use of blood and tissue samples, as well as the subsequent analyses. The patients received no financial compensation or gifts.

\section{Consent for publication}

Not applicable.

\section{Competing interests}

The authors declare that they have no competing interests.

\section{Publisher's Note}

Springer Nature remains neutral with regard to jurisdictional claims in published maps and institutional affiliations.

\section{Author details}

${ }^{1}$ Department of Pathology, Fujian Cancer Hospital \& Fujian Medical University Cancer Hospital, No.420 Fu Ma Road, Jin An District, Fuzhou 350014, Fujian, China. ${ }^{2}$ Department of Cardiology, The First Affiliated Hospital of Fujian Medical University, Fuzhou, Fujian, China. ${ }^{3}$ Department of Medical Record, Fujian Cancer Hospital \& Fujian Medical University Cancer Hospital, Fuzhou, Fujian, China. ${ }^{4}$ Department of Core Research Laboratory, Fujian Cancer Hospital \& Fujian Medical University Cancer Hospital, Fuzhou, Fujian, China. ${ }^{5}$ Institute of Clinical Medical Sciences, China-Japan Friendship Hospital, No.2 Yinghua East Street, Chao Yang District, Beijing 100029, China.

\section{Received: 23 August 2017 Accepted: 14 June 2018}

\section{Published online: 06 August 2018}

\section{References}

1. Plummer M, Franceschi S, Vignat J, Forman D, de Martel C. Global burden of gastric cancer attributable to helicobacter pylori. Int $J$ Cancer. 2015:136(2):487-90.

2. Chen W, Zheng R, Baade PD, Zhang S, Zeng H, Bray F, Jemal A, Yu XQ, He J. Cancer statistics in China, 2015. CA Cancer J Clin. 2016:66(2):115-32.

3. Bertuccio P, Chatenoud L, Levi F, Praud D, Ferlay J, Negri E, Malvezzi M, La Vecchia C. Recent patterns in gastric cancer: a global overview. Int J Cancer. 2009;125(3):666-73.

4. Park YM, Kim JH, Baik SJ, Park JJ, Youn YH, Park H. Clinical risk assessment for gastric cancer in asymptomatic population after a health check-up: an individualized consideration of the risk factors. Medicine (Baltimore). 2016; 95(44):e5351.

5. Park JY, Kim YW, Ryu KW, Nam BH, Lee YJ, Jeong SH, Park JH, Hur H, Han SU, Min JS, et al. Assessment of laparoscopic stomach preserving surgery with sentinel basin dissection versus standard gastrectomy with lymphadenectomy in early gastric cancer-a multicenter randomized phase III clinical trial (SENORITA trial) protocol. BMC Cancer. 2016;16:340.

6. Torre LA, Bray F, Siegel RL, Ferlay J, Lortet-Tieulent J, Jemal A. Global cancer statistics, 2012. CA Cancer J Clin. 2015;65(2):87-108.

7. Feng F, Zheng G, Guo X, Liu Z, Xu G, Wang F, Wang Q, Guo M, Lian X, Zhang $\mathrm{H}$. Impact of body mass index on surgical outcomes of gastric cancer. BMC Cancer. 2018;18(1):151

8. Yu X, Hu F, Yao Q, Li C, Zhang H, Xue Y. Serum fibrinogen levels are positively correlated with advanced tumor stage and poor survival in patients with gastric cancer undergoing gastrectomy: a large cohort retrospective study. BMC Cancer. 2016;16:480.
9. Hu D, Peng F, Lin X, Chen G, Zhang H, Liang B, Ji K, Lin J, Chen LF, Zheng $X$, et al. Preoperative metabolic syndrome is predictive of significant gastric cancer mortality after gastrectomy: the Fujian prospective investigation of cancer (FIESTA) study. EBioMedicine. 2017:15:73-80.

10. Eliasson B, Cederholm J, Eeg-Olofsson K, Svensson AM, Zethelius B, Gudbjornsdottir S, National Diabetes R. Clinical usefulness of different lipid measures for prediction of coronary heart disease in type 2 diabetes: a report from the Swedish National Diabetes Register. Diabetes Care. 2011; 34(9):2095-100

11. Park HR, Shin SR, Han AL, Jeong YJ. The correlation between the triglyceride to high density lipoprotein cholesterol ratio and computed tomographymeasured visceral fat and cardiovascular disease risk factors in local adult male subjects. Korean J Fam Med. 2015;36(6):335-40.

12. Hu D, Peng F, Lin X, Chen G, Liang B, Li C, Zhang H, Liao X, Lin J, Zheng X, et al. The elevated preoperative fasting blood glucose predicts a poor prognosis in patients with esophageal squamous cell carcinoma: the Fujian prospective investigation of cancer (FIESTA) study. Oncotarget. 2016;7(40):65247-56.

13. Hu D, Lin X, Chen Y, Chang Q, Chen G, Li C, Zhang H, Cui Z, Liang B, Jiang $W$, et al. Preoperative blood-routine markers and prognosis of esophageal squamous cell carcinoma: the Fujian prospective investigation of cancer (FIESTA) study. Oncotarget. 2017;8(4):23841-50.

14. Peng F, Hu D, Lin X, Chen G, Liang B, Zhang H, Dong X, Lin J, Zheng X, Niu $W$. Analysis of preoperative metabolic risk factors affecting the prognosis of patients with esophageal squamous cell carcinoma: the Fujian prospective investigation of cancer (FIESTA) study. EBioMedicine. 2017;16:115-23.

15. Sha H, Hu D, Wu S, Peng F, Xu G, Fan G, Lin X, Chen G, Liang B, Chen Y, et al. Baseline metabolic risk score and postsurgical esophageal cancerspecific mortality: the Fujian prospective investigation of cancer (FIESTA) study. J Cancer. 2018;9(7):1173-81.

16. Hu D, Zhang H, Lin X, Chen G, Li C, Liang B, Chen Y, Cui Z, Peng F, Zheng $X$, et al. Elevated preoperative neutrophil-to-lymphocyte ratio can predict poor survival in early stage gastric cancer patients receiving radical gastrectomy: the Fujian prospective investigation of cancer (FIESTA) study. J Cancer. 2017;8(7):1214-22.

17. Peng F, Hu D, Lin X, Chen G, Liang B, Zhang H, Ji K, Huang J, Lin J, Zheng $X$, et al. Preoperative metabolic syndrome and prognosis after radical resection for colorectal cancer: the Fujian prospective investigation of cancer (FIESTA) study. Int J Cancer. 2016;139(12):2705-13.

18. Peng F, Hu D, Lin $X$, Chen G, Li C, Chen Y, Liang B, Cui Z, Zhang H, Lin J, et al. The monocyte to red blood cell count ratio is a strong predictor of postoperative survival in colorectal cancer patients: the Fujian prospective investigation of cancer (FIESTA) study. J Cancer. 2017:8(6):967-75.

19. Peng F, Hu D, Lin X, Chen G, Liang B, Chen Y, Li C, Zhang H, Xia Y, Lin J, et al. An in-depth prognostic analysis of baseline blood lipids in predicting postoperative colorectal cancer mortality: the FIESTA study. Cancer Epidemiol. 2018:52:148-57.

20. Peng F, Hu D, Lin X, Liang B, Chen Y, Zhang H, Xia Y, Lin J, Zheng X, Niu W Impact of long-term antihypertensive and antidiabetic medications on the prognosis of post-surgical colorectal cancer: the Fujian prospective investigation of cancer (FIESTA) study. Aging (Albany NY). 2018:10(5):1166-81.

21. Perloff D, Grim C, Flack J, Frohlich ED, Hill M, McDonald M, Morgenstern BZ. Human blood pressure determination by sphygmomanometry. Circulation. 1993;88(5 Pt 1):2460-70

22. Edge SB, Compton CC. The American Joint Committee on Cancer: the 7th edition of the AJCC cancer staging manual and the future of TNM. Ann Surg Oncol. 2010;17(6):1471-4.

23. Vickers AJ, Elkin EB. Decision curve analysis: a novel method for evaluating prediction models. Med Decis Mak. 2006;26(6):565-74

24. Pu X, Ye $Y$, Wu X. Development and validation of risk models and molecular diagnostics to permit personalized management of cancer. Cancer. 2014:120(1):11-9.

25. Xiao S, Zhou L. Gastric cancer: metabolic and metabolomics perspectives (review). Int J Oncol. 2017:51:5-17.

26. Lin Y, Ness-Jensen E, Hveem K, Lagergren J, Lu Y. Metabolic syndrome and esophageal and gastric cancer. Cancer Causes Control. 2015:26(12):1825-34.

27. Lindkvist B, Almquist M, Bjorge T, Stocks T, Borena W, Johansen D, Hallmans G, Engeland A, Nagel G, Jonsson $H$, et al. Prospective cohort study of metabolic risk factors and gastric adenocarcinoma risk in the Metabolic Syndrome and Cancer Project (Me-Can). Cancer Causes Control. 2013;24(1):107-16. 
28. Pourhoseingholi MA, Ashtari S, Hajizadeh N, Zali MR. Metabolic syndrome, gastric cancer mortality and competing risk survival analysis. EBioMedicine. 2017;15:4-5

29. Lin L, Huang H, Liao W, Ma H, Liu J, Wang L, Huang N, Liao Y, Liao W. MACC1 supports human gastric cancer growth under metabolic stress by enhancing the Warburg effect. Oncogene. 2015;34(21):2700-10.

30. Wei XL, Qiu MZ, Lin HX, Zhang Y, Liu JX, Yu HM, Liang WP, Jin Y, Ren C, He MM, et al. Patients with old age or proximal tumors benefit from metabolic syndrome in early stage gastric cancer. PLoS One. 2014;9(3):e89965.

31. Kim EH, Lee H, Chung H, Park JC, Shin SK, Lee SK, Hyung WJ, Lee YC, Noh SH. Impact of metabolic syndrome on oncologic outcome after radical gastrectomy for gastric cancer. Clin Res Hepatol Gastroenterol. 2014;38(3):372-8.

32. Chang WC, Huang SF, Lee YM, Lai HC, Cheng BH, Cheng WC, Ho JY, Jeng LB, Ma WL. Cholesterol import and steroidogenesis are biosignatures for gastric cancer patient survival. Oncotarget. 2017:8(1):692-704.

33. Ock CY, Kim TY, Lee KH, Han SW, Im SA, Bang YJ, Oh DY. Metabolic landscape of advanced gastric cancer according to HER2 and its prognostic implications. Gastric Cancer. 2016;19(2):421-30.

34. Grundy SM, Bilheimer D, Chait A, Clark LT, Denke M, Havel RJ, Hazzard WR, Hulley SB, Hunninghake DB, Kreisberg RA, Etherton PK, McKenney JM, Newman MA, Schaefer EJ, Sobel BE, Somelofski C, Weinstein MC, Brewer Jr $H B$, Cleeman Jl, Donato KA, Ernst N, Hoeg JM, Rifkind BM, Rossouw J, Sempos CT, Gallivan JM, Harris MN, Quint-Adler L. Summary of the second report of the National Cholesterol Education Program (NCEP) expert panel on detection, evaluation, and treatment of high blood cholesterol in adults (adult treatment panel II). JAMA. 1993;269(23):3015-23.

35. Association C. The suggestion on Chinese metabolic syndrome. Shanghai: Chinese Medical Association; 2004.

36. His M, Zelek L, Deschasaux M, Pouchieu C, Kesse-Guyot E, Hercberg S, Galan P, Latino-Martel P, Blacher J, Touvier M. Prospective associations between serum biomarkers of lipid metabolism and overall, breast and prostate cancer risk. Eur J Epidemiol. 2014;29(2):119-32.

37. Wulaningsih W, Garmo H, Holmberg L, Hammar N, Jungner I, Walldius G, Van Hemelrijck M. Serum lipids and the risk of gastrointestinal malignancies in the Swedish AMORIS study. J Cancer Epidemiol. 2012;2012:792034.

38. Prosser HC, Tan JT, Dunn LL, Patel S, Vanags LZ, Bao S, Ng MK, Bursill CA. Multifunctional regulation of angiogenesis by high-density lipoproteins. Cardiovasc Res. 2014;101(1):145-54.

39. Brusselaers N, Maret-Ouda J, Konings P, El-Serag HB, Lagergren J. Menopausal hormone therapy and the risk of esophageal and gastric cancer. Int J Cancer. 2017;140(7):1693-9.

40. Zhang XJ, Xiao Z, Yu HL, Zhang XX, Cheng Z, Tian HM. Short-term glucose metabolism and gut hormone modulations after Billroth II gastrojejunostomy in nonobese gastric cancer patients with type 2 diabetes mellitus, impaired glucose tolerance and normal glucose tolerance. Arch Med Res. 2013;44(6):437-43.

\section{Ready to submit your research? Choose BMC and benefit from:}

- fast, convenient online submission

- thorough peer review by experienced researchers in your field

- rapid publication on acceptance

- support for research data, including large and complex data types

- gold Open Access which fosters wider collaboration and increased citations

- maximum visibility for your research: over $100 \mathrm{M}$ website views per year

At BMC, research is always in progress.

Learn more biomedcentral.com/submissions 